\title{
Magnetic monopole search at high altitude with the SLIM experiment
}

\author{
S. Balestra ${ }^{1,2}$, S. Cecchini ${ }^{1,3}$, M. Cozzi ${ }^{1,2}$, M. Errico ${ }^{1,2}$, F. Fabbri ${ }^{2}$, G. Giacomelli ${ }^{1,2}$, R. Giacomelli ${ }^{2}$,

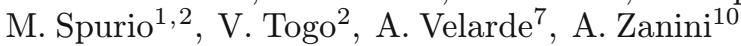 \\ ${ }^{1}$ Dip. Fisica dell'Universitá di Bologna, 40127 Bologna, Italy \\ 2 INFN Sez. Bologna, Viale C. Berti Pichat 6/2, 40127 Bologna, Italy \\ ${ }^{3}$ INAF/IASF Sez. Bologna, 40129 Bologna, Italy \\ ${ }^{4}$ Physics Dept., Sant Longowal Institute of Eng. \& Tech., Longowal, 148 106, India \\ 5 PD, PINSTECH, P.O. Nilore, and COMSATS-CIIT, No. 30, H-8/1, Islamabad, Pakistan \\ ${ }^{6}$ Centre for Subatomic Research, Univ. of Alberta, Edmonton, Alberta T6G 2N4, Canada \\ 7 Laboratorio de Física Cósmica de Chacaltaya, UMSA, La Paz, Bolivia \\ ${ }^{8}$ Institute for Space Sciences, 077125 Bucharest-Măgurele, Romania \\ ${ }^{9}$ Dip. Fisica Sperimentale e Generale, Universitá di Torino, 10125 Torino, Italy \\ 10 INFN Sez. Torino, 10125 Torino, Italy \\ 11 Astrophysics Dept., CRAAG, BP 63 Bouzareah, 16340 Algiers, Algeria
} M. Giorgini ${ }^{1,2}$, a , A. Kumar ${ }^{1,4}$, S. Manzoor ${ }^{1,5}$, J. McDonald ${ }^{6}$, G. Mandrioli ${ }^{2}$, S. Marcellini ${ }^{2}$, A. Margiotta ${ }^{1,2}$, E. Medinaceli ${ }^{1,7}$, L. Patrizii ${ }^{2}$, J. Pinfold ${ }^{6}$, V. Popa ${ }^{2,8}$, I.E. Qureshi ${ }^{5}$, O. Saavedra ${ }^{9}, 10$, Z. Sahnoun ${ }^{2,11}$, G. Sirri ${ }^{2}$,

Received: 19 February 2008 / Revised version: 21 March 2008 /

Published online: 19 April 2008 - (C) Springer-Verlag / Società Italiana di Fisica 2008

\begin{abstract}
The SLIM experiment was a large array of nuclear track detectors located at the Chacaltaya high altitude Laboratory (5230 m a.s.l.). The detector was in particular sensitive to intermediate mass magnetic monopoles, with masses $10^{5} \mathrm{GeV}<M_{M}<10^{12} \mathrm{GeV}$. From the analysis of the full detector exposed for more than 4 years a flux upper limit of $1.3 \times 10^{-15} \mathrm{~cm}^{-2} \mathrm{~s}^{-1} \mathrm{sr}^{-1}$ for downgoing fast intermediate mass monopoles was established at the $90 \%$ C.L.
\end{abstract}

PACS. 14.80.Hv; 29.40.Wk; 29.90.+r

\section{Introduction}

In 1931 Dirac introduced magnetic monopoles (MMs) in order to explain the quantization of the electric charge, obtaining the formula $e g=n \hbar c / 2$, from which $g=n g_{\mathrm{D}}=$ $n \hbar c / 2 e=n 68.5 e=n 3.29 \times 10^{-8}$ in the c.g.s. symmetric system of units [1]; $n$ is an integer, $n=1,2,3, \ldots$ MMs possessing an electric charge and bound systems of a magnetic monopole with an atomic nucleus are called dyons. An extensive bibliography on MMs is given in [2]. Relatively low mass classical Dirac monopoles have been searched for at high energy accelerators [3-8].

Magnetic monopoles are present in a variety of unified gauge models with a wide range of masses.

Grand unified theories (GUT) of the strong and electroweak interactions at the mass scale $M_{G} \sim 10^{14}-10^{15} \mathrm{GeV}$ predict the existence of magnetic monopoles, produced in the early Universe at the end of the GUT epoch, with very large masses, $M_{M} \geq 10^{16} \mathrm{GeV}$. Such monopoles cannot be produced with existing accelerators, nor

\footnotetext{
a e-mail: miriam.giorgini@bo.infn.it
}

with any foreseen for the future. In the past, GUT poles were searched for in the cosmic radiation. These poles are characterized by low velocities and relatively large energy losses [9-13]. The MACRO experiment set the best limits on GUT MMs with $g=g_{\mathrm{D}}, 2 g_{\mathrm{D}}, 3 g_{\mathrm{D}}$ and dyons at the level of $\sim 1.4 \times 10^{-16} \mathrm{~cm}^{-2} \mathrm{~s}^{-1} \mathrm{sr}^{-1}$ for $4 \times 10^{-5}<\beta=$ $v / c<0.7[14-17]$.

Some GUT models and some supersymmetric models predict intermediate mass monopoles (IMMs) with masses $10^{5} \mathrm{GeV}<M_{M}<10^{12} \mathrm{GeV}$ and with magnetic charges of multiples of $g_{\mathrm{D}}$; these MMs may have been produced in later phase transitions in the early Universe and could be present in the cosmic radiation [18-22].

IMMs may be relativistic since they could be accelerated to high velocities in one coherent domain of the galactic magnetic field. In this case one would have to look for downgoing, fast $(\beta>0.03)$, heavily ionizing $\mathrm{MMs}^{1}$.

The main purpose of the SLIM (search for light monopoles) experiment at the Chacaltaya laboratory in Bolivia

\footnotetext{
1 The interest in MMs was also connected with the possibility
} that they could yield the highest energy cosmic rays [21, 22]. 


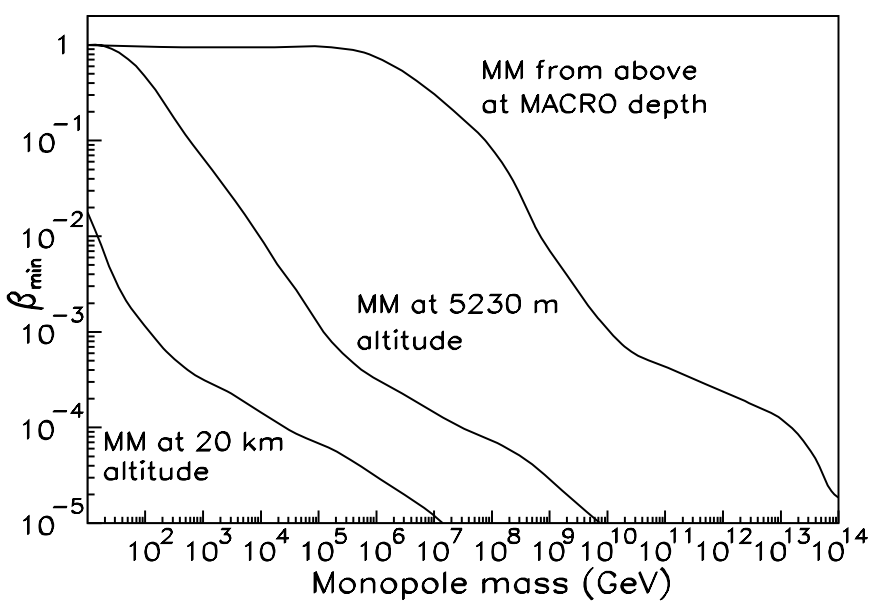

Fig. 1. Accessible regions (above lines) in the plane (mass, $\beta$ ) for monopoles with magnetic charge $g=g_{D}$ coming from above for an experiment at altitudes of $20000 \mathrm{~m}, 5230 \mathrm{~m}$, and for an underground detector at the Gran Sasso Lab. (average rock overburden of 3700 m.w.e.)

at $5230 \mathrm{~m}$ a.s.l., was the search for IMMs [23,24]. An exposure at a high altitude laboratory allows to search for MMs of lower masses, higher magnetic charges and lower velocities, see Fig. 1.

The searches for IMMs by Earth based detectors are essentially limited to downgoing particles $[25,26]$. Water Cherenkov detectors are limited to fast downgoing IMMs (with $\beta>0.5$ ), and a search can be done if the detectors are able to discriminate against the large background of cosmic ray muons [27].

The SLIM detector was also sensitive to strange quark matter nuggets [28-33] and Q-balls [34-36]. The results on these dark matter candidates will be discussed elsewhere.

In the following, we present a short description of the SLIM apparatus, the calibrations of the nuclear track detectors (NTDs), the etching and analysis procedures, and the limits obtained by the experiment on IMMs and GUT magnetic monopoles.

\section{Experimental procedure}

The SLIM experiment was an array of NTDs ${ }^{2}$ with a total surface area slightly greater than $400 \mathrm{~m}^{2}[23,24]$. The array was organized into 7410 modules, each of area $24 \times 24 \mathrm{~cm}^{2}$. All modules were made up of: three layers of CR39 ${ }^{\circledR 3}$, each $1.4 \mathrm{~mm}$ thick; 3 layers of Makrofol $\mathrm{DE}^{\circledR 4}$, each $0.48 \mathrm{~mm}$ thick; 2 layers of Lexan each $0.25 \mathrm{~mm}$ thick and one layer of aluminum absorber $1 \mathrm{~mm}$ thick (see Fig. 2 right). The

\footnotetext{
2 Another $100 \mathrm{~m}^{2}$ of NTDs were installed at Koksil (Pakistan, $4275 \mathrm{~m}$ a.s.l.) since 2002 and were not used in the present analysis.

3 The SLIM CR39 was produced by the Intercast Europe Co., Parma, Italy according to our specifications.

4 Manufactured by Bayer AG, Leverkusen, Germany.
}

CR39 used in about $90 \%$ of the modules $\left(377 \mathrm{~m}^{2}\right)$ was of the same type used in the MACRO experiment [14-17]. The remaining modules, $50 \mathrm{~m}^{2}$, utilized CR39 containing $0.1 \%$ of DOP additive, CR39(DOP).

Each module (stack) was sealed in an aluminized plastic bag $(125 \mu \mathrm{m}$ thick) filled with dry air at a pressure of 1 bar. The modules were transported to La Paz, Bolivia, from Italy in wooden boxes and their position with respect to the other modules in the shipping crate was recorded. The stacks were deployed under the roof of the Chacaltaya Laboratory, roughly $4 \mathrm{~m}$ above ground (see Fig. 2 left). The installation of the SLIM detectors started in February 2000 and ended in February 2002. The return of the material to Italy was organized in batches, after the completion of the 4 years exposure.

The atmospheric pressure at Chacaltaya is about 0.5 bar; before shipping to Chacaltaya, in Bologna we checked the air tightness of the envelopes sealed with air at a pressure of 1 bar by placing a sample of them in an airtight tank at a pressure of $0.3 \mathrm{~atm}$ for a few months; no significant leakage was detected.

From the experience gained with the MACRO Nuclear Track Subdetector [14-17], we know that the used CR39 does not suffer from "aging" or "fading" effects for exposure times as long as 10 years [37]. Further calibrations with $1 \mathrm{~A} \mathrm{GeV} \mathrm{Fe}{ }^{26+}$ ions in 1999 and 2005 and with $158 \mathrm{~A} \mathrm{GeV} \mathrm{In}^{49+}$ in 2003 confirmed the quality and the stability of the CR39 used in the SLIM experiment [38-41].

\subsection{Environmental measurements}

During the first phases of the detector deployment we evaluated possible effects of climatic conditions on the detector response and possible backgrounds. Previous tests had shown that the CR39 response does not depend on the time elapsed from its production and the passage of the particle if the ambient temperature ranges between $-20^{\circ} \mathrm{C}$ and $+30^{\circ} \mathrm{C}$. The minimum and maximum values of the air temperature in each detector hall in Chacaltaya was recorded 3 times a day over the lifetime of the experiment. The temperature values usually ranged from 0 to $30^{\circ} \mathrm{C}$ with an average value of $12^{\circ} \mathrm{C}$ for the whole year and from one year to the other; however in the summer months in very few cases temperatures down to $-5^{\circ} \mathrm{C}$ were measured in the early morning. Therefore, no significant variations were expected in the detector response over the exposure period.

We performed measurements of the radon concentration in different locations of the experimental rooms where the SLIM detectors were placed. We used for this purpose E-PERM ${ }^{\circledR}$ radon dosimeters. The measured radon activity was about $40-50 \mathrm{~Bq} / \mathrm{m}^{3}$ of air. According to our previous experience with the MACRO NTDs, we concluded that this level of radon induced radioactivity did not present a problem for the experiment, even in case of radon diffusion into the module bags.

Two different types of neutron detectors (BTI bubble counters and a BF3 counter detectors) were used to measure the neutron flux at Chacaltaya, during the first instal- 


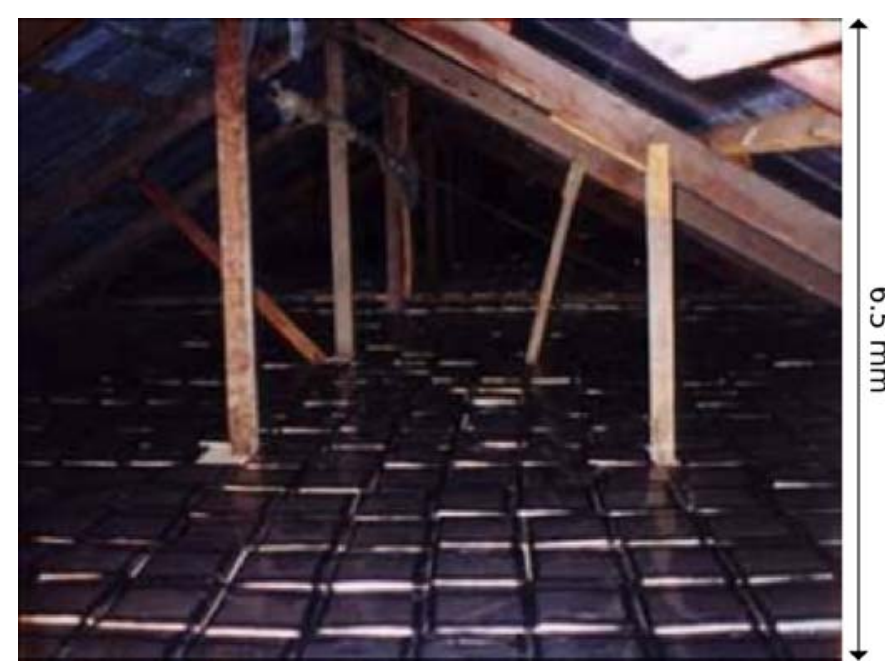

lation shift of 2001 over the energy range of a few hundred $\mathrm{keV}$ to about $20 \mathrm{MeV}[42,43]$. Neutrons of these energies interacting inside the detectors could induce background tracks, and their density could affect the scanning speed and efficiency. Both types of neutron detectors measured the accumulated dose. Consistent results were obtained by both types of detectors. The accumulated dose measured in open air and near the detectors was very similar. The absolute neutron flux was computed using the BTI bubble counters for which the efficiency is known. A value of $(1.7 \pm 0.8) \times 10^{-2} \mathrm{~cm}^{-2} \mathrm{~s}^{-1}$ was obtained, which is in agreement with other reported neutron flux data at the altitude of Chacaltaya and with more recent measurements at the same location [44]. The necessity to reduce the neutron induced background in CR39 required us to study special etching procedures, mainly based on the addition of ethyl alcohol to the etching solutions. As discussed in the next section, the addition of alcohol reduces the background tracks on the detector sheets and improves the surface quality (i.e. greater transparency), at the expense of a higher threshold [38-41].

\subsection{Etching procedures}

The passage of a magnetic monopole in NTDs, such as CR39, is expected to cause structural line damage in the polymer (forming the so called "latent track"). Since IMMs have a constant energy loss through the stacks, the subsequent chemical etching should result in collinear etch-pit cones of equal size on both faces of each detector sheet. In order to increase the detector "signal to noise" ratio different etching conditions [37-41] were defined. The so-called "strong etching" technique allows better surface quality and larger post-etched cones to be obtained. This makes etch pits easier to detect under visual scanning. Strong etching was used to analyze the top-most CR39 sheet in each module. "Soft etching" was applied to the other CR39 layers in a module if a candidate track was found after the first scan. This process allows to proceed in several etching steps and study the formation of the post-etched cones.

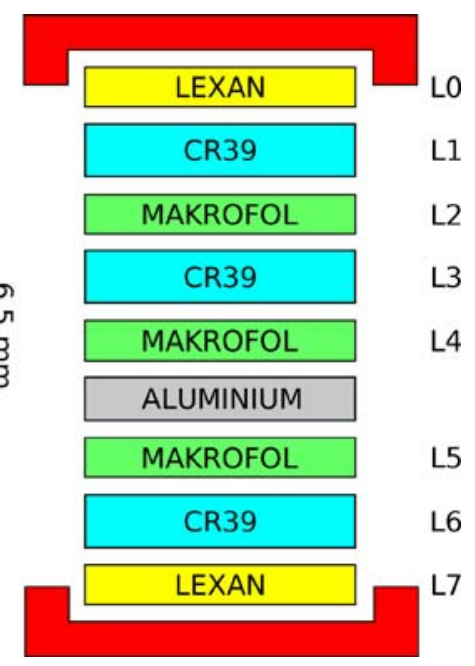

Fig. 2. Left: the SLIM modules installed at Chacaltaya. Right: composition of one of the 7410 modules; each module was enclosed in an aluminized mylar bag filled with dry air at a pressure of 1 bar
For CR39 and CR39(DOP) the strong etching conditions were: $8 \mathrm{~N} \mathrm{KOH}+1.5 \%$ ethyl alcohol at $75^{\circ} \mathrm{C}$ for $30 \mathrm{~h}$. The bulk etching velocities were $v_{\mathrm{B}}=7.2 \pm 0.4 \mu \mathrm{m} / \mathrm{h}$ and $v_{\mathrm{B}}=5.9 \pm 0.3 \mu \mathrm{m} / \mathrm{h}$ for CR39 and CR39(DOP), respectively.

The soft etching conditions were $6 \mathrm{~N} \mathrm{NaOH}+1 \%$ ethyl alcohol at $70^{\circ} \mathrm{C}$ for $40 \mathrm{~h}$ for CR39 and CR39(DOP). The bulk etching rates were $v_{\mathrm{B}}=1.25 \pm 0.02 \mu \mathrm{m} / \mathrm{h}$ and $v_{\mathrm{B}}=0.98 \pm 0.02 \mu \mathrm{m} / \mathrm{h}$ for CR39 and CR39(DOP), respectively.

Makrofol NTDs were etched in $6 \mathrm{~N} \mathrm{KOH}+20 \%$ ethyl alcohol at $50{ }^{\circ} \mathrm{C}$ for $10 \mathrm{~h}$; the bulk etch velocity was $v_{\mathrm{B}}=3.4 \mu \mathrm{m} / \mathrm{h}$.

\subsection{NTD calibrations}

The CR39 and Makrofol nuclear track detectors were calibrated with $158 \mathrm{~A} \mathrm{GeV} \mathrm{In}^{49+}$ and $\mathrm{Pb}^{82+}$ beams at the CERN SPS and $1 \mathrm{~A} \mathrm{GeV} \mathrm{Fe}{ }^{26+}$ at the Brookhaven National Laboratory (BNL) Alternating Gradient Synchrotron (AGS). The calibration layout was a standard one with a fragmentation target and CR39 (plus Makrofol) NTDs in front of and behind the target [45]. The detector sheets behind the target detected both primary ions and nuclear fragments of decreasing charge.

We recall that the formation of etch-pit cones ("tracks") in NTDs is regulated by the bulk etching rate, $v_{\mathrm{B}}$, and the track etching rate, $v_{\mathrm{T}}$, i.e. the velocities at which the undamaged and damaged materials (along the particle trajectory), are etched out. Etch-pit cones are formed if $v_{\mathrm{T}}>v_{\mathrm{B}}$. The response of the CR39 detector is measured by the etching rate ratio $p=v_{\mathrm{T}} / v_{\mathrm{B}}$.

After etching the standard calibration procedure was the following:

1. Measure the base area of each track in NTDs with an automatic image analyzer system [46]. The projectile fragments carry the same $\beta$ and approximately the same direction of the incident ion; the $Z$ of each resolved peak is identified via the base area spectrum. The average base area distributions of the $\mathrm{In}^{49+}$ ions and of their fragments 

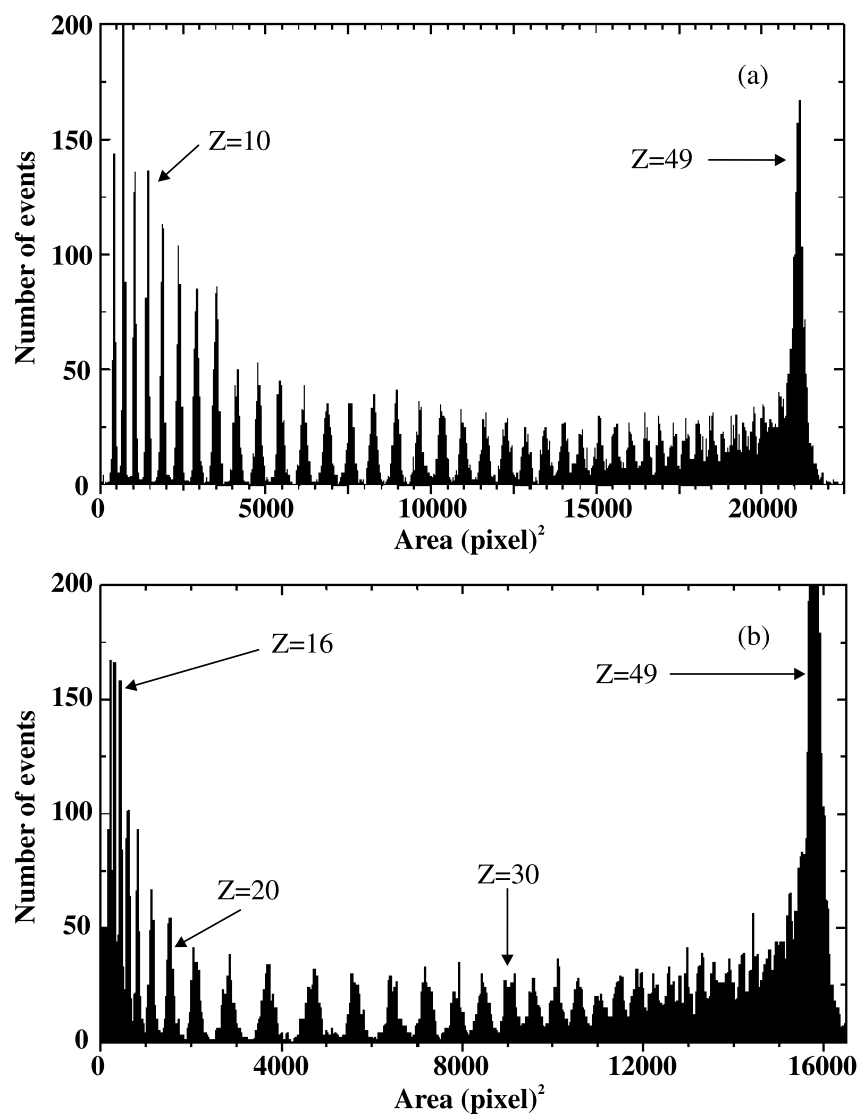

Fig. 3. Calibrations of CR39 nuclear track detectors with $158 \mathrm{~A} \mathrm{GeV} \mathrm{In}^{49+}$ ions and their nuclear fragments with decreasing charge. The base areas $\left(1\right.$ pixel $\left.^{2}=0.3 \mu \mathrm{m}^{2}\right)$ of the etched cones were averages over 2 faces. The CR39 was etched in a soft and $\mathbf{b}$ strong etching conditions

in CR39, etched in soft or strong conditions, are shown in Fig. 3a and $\mathrm{b}\left(1\right.$ pixel $\left.^{2}=0.3 \mu \mathrm{m}^{2}\right)$.

2. For each calibration peak the $Z / \beta$ is obtained and the reduced etch rate $(p-1)$ is computed. The restricted

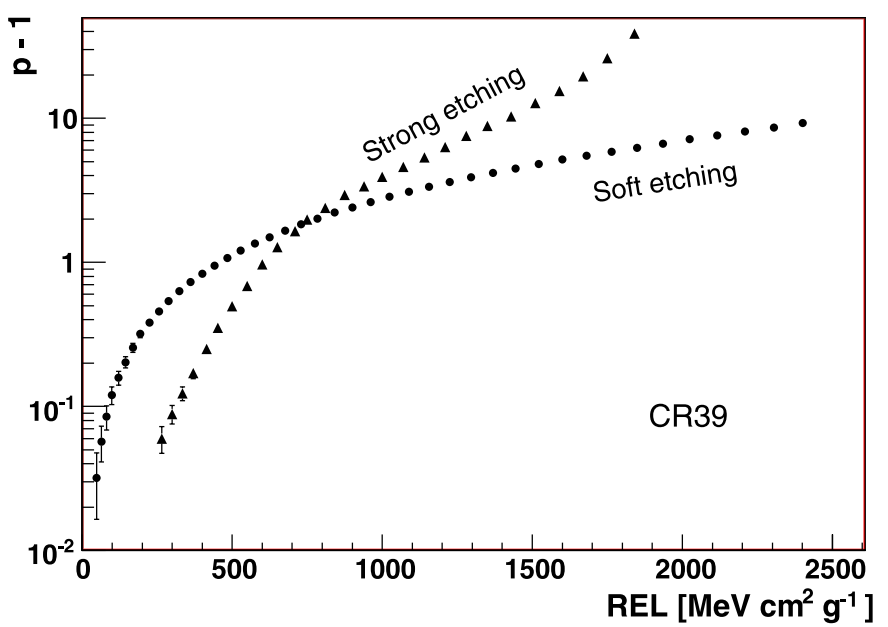

energy loss (REL) due to ionization and nuclear scattering is evaluated, thus arriving to the calibration data of $(p-1)$ vs. REL shown in Fig. 4 for both strong and soft etching conditions for CR39 and CR39(DOP). For soft etching the threshold in CR39 is at $Z / \beta \sim 7$ corresponding to REL $\sim 50 \mathrm{MeV} \mathrm{cm}{ }^{2} \mathrm{~g}^{-1}$. For strong etching the threshold is at $Z / \beta \sim 14$, corresponding to $\mathrm{REL} \sim 200 \mathrm{MeV} \mathrm{cm}^{2} \mathrm{~g}^{-1}$. The extrapolation of the calibration curves to $p=1$ gives REL $\lesssim 40 \mathrm{MeV} \mathrm{cm}^{2} \mathrm{~g}^{-1}$

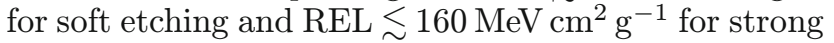
etching. For CR39(DOP) the threshold in soft etching conditions is at $Z / \beta \sim 13$ corresponding to REL $\sim$ $170 \mathrm{MeV} \mathrm{cm}^{2} \mathrm{~g}^{-1}$; the threshold in strong etching conditions is at $Z / \beta \sim 21$ corresponding to REL $460 \mathrm{MeV} \mathrm{cm}{ }^{2} \mathrm{~g}^{-1}$. The extrapolation of the calibration curves to $p=1$ gives REL $\lesssim 240 \mathrm{MeV} \mathrm{cm}^{2} \mathrm{~g}^{-1}$ for strong etching.

For magnetic monopoles with $g=g_{\mathrm{D}}, 2 g_{\mathrm{D}}, 3 g_{\mathrm{D}}$ we computed the REL as a function of $\beta$ taking into account electronic and nuclear energy losses, see Fig. 5 [47].

With the used etching conditions, the CR39 allows the detection of

1. MMs with $g=g_{\mathrm{D}}$ for $\beta \sim 10^{-4}$ and for $\beta>10^{-2}$;

2. MMs with $g=2 g_{\mathrm{D}}$ for $\beta$ around $10^{-4}$ and for $\beta>$ $4 \times 10^{-3}$;

3. The whole $\beta$-range of $4 \times 10^{-5}<\beta<1$ is accessible for MMs with $g>2 g_{\mathrm{D}}$ and for dyons.

For the Makrofol polycarbonate the detection threshold is at $Z / \beta \sim 50$ and $\mathrm{REL} \sim 2.5 \mathrm{GeV} \mathrm{cm}^{2} \mathrm{~g}^{-1}$ [38-41]; for this reason the use of Makrofol is restricted to the search for fast MMs.

\subsection{Analysis}

After exposure at Chacaltaya the modules were brought back by air flights to Italy in order to be etched and analyzed in the Bologna laboratory. Three "reference" holes of $2 \mathrm{~mm}$ diameter were drilled in each module with a precision machine (the hole locations were defined to

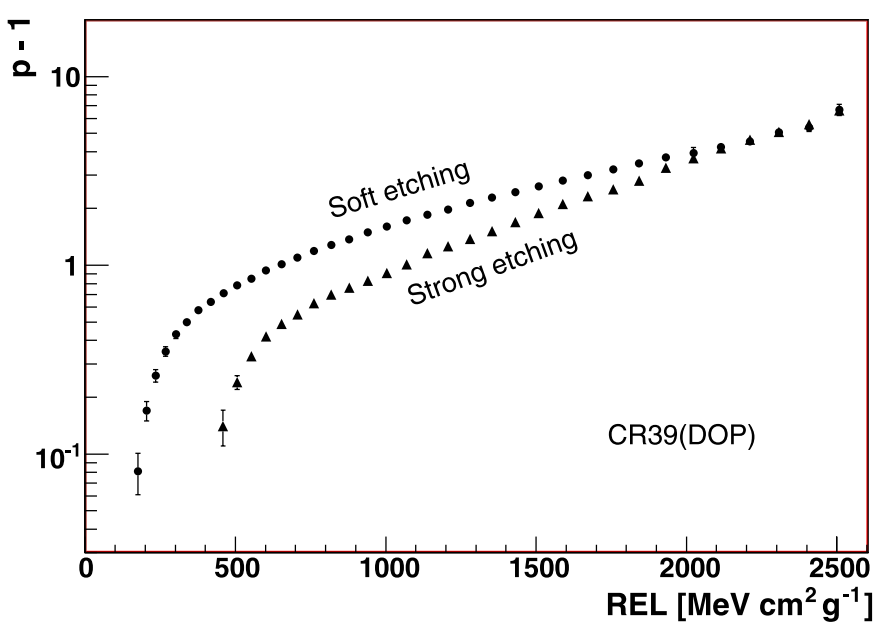

Fig. 4. Reduced track etch rate $(p-1)$ vs. REL for the CR39 (left) and CR39(DOP) (right) detectors, exposed to the $158 \mathrm{~A}$ GeV indium ion beam, etched in soft and strong etching conditions 


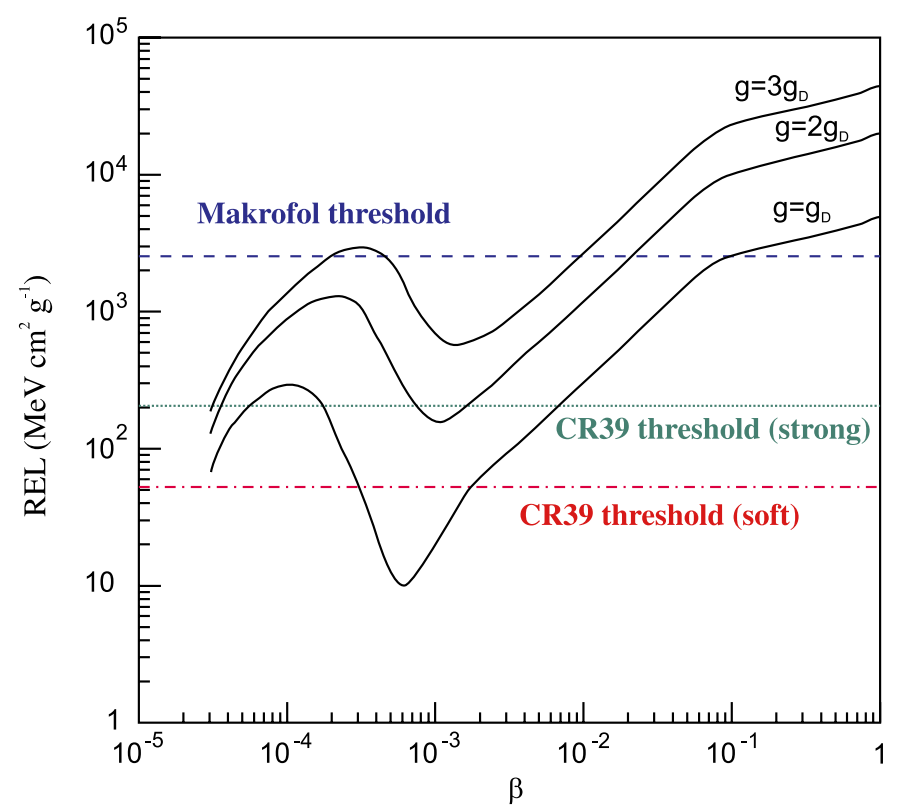

Fig. 5. REL vs. beta for magnetic monopoles with $g=g_{\mathrm{D}}$, $2 g_{\mathrm{D}}, 3 g_{\mathrm{D}}$. The dashed lines represent the CR39 thresholds in soft and strong etching conditions and the Makrofol threshold (see Sect. 2.2)

within $100 \mu \mathrm{m})$. This allowed us to follow the passage of a "candidate" through the stack. The bags (envelopes) were opened, the detectors were labeled and their thicknesses were measured, using a micrometer, in 9 uniformly distributed points on the foil surface.

The analysis of a SLIM module started by etching the uppermost CR39 sheet using strong conditions in order to reduce the CR39 thickness from 1.4 to $\sim 0.9 \mathrm{~mm}$. After the strong etching, the CR39 sheet was scanned twice, with a stereo microscope, by different operators, with a $3 \times$ magnification optical lens, looking for any possible correspondence of etch pits on the two opposite surfaces. The measured single scan efficiency was about $99 \%$; thus the double scan guarantees an efficiency of $\sim 100 \%$ for finding a possible signal.

Further observation of a "suspicious correspondence" was made with an optical $20-40 \times$ stereo microscope and classified either as a defect or a candidate track. This latter was then examined by an optical microscope with $6.3_{\mathrm{ob}} \times$ $25_{\text {oc }}$ magnification and the axes of the base-cone ellipses in the front and back sides were measured.

A track was defined as a "candidate" if the computed $p$ and incident angle $\theta$ on the front and back sides were equal to within $20 \%$. For each candidate the azimuth angle $\varphi$ and its position $P$ referred to the fiducial marks were also determined. The uncertainties $\Delta \theta, \Delta \varphi$ and $\Delta P$ defined a "coincidence" area $\left(<0.5 \mathrm{~cm}^{2}\right)$ around the candidate expected position in the other layers, as shown in Fig. 6 .

In this case the lowermost CR39 layer was etched in soft etching conditions, and an accurate scan under an optical microscope with high magnification $(500 \times$ or $1000 \times)$ was performed in a square region around the candidate expected position, which included the "coincidence" area. If a two-fold coincidence was detected, the CR39 middle layer was also analyzed.

The bottom CR39 sheet was etched in about 50 cases; the third CR39 sheet was etched only in few cases, when there was still a possible uncertainty, and for checks ( $\sim 16$ times). Some Makrofol foils were etched for reasons similar to the previous point and for other checks concerning the Makrofol itself ( $\sim 12$ times $)$.

\section{Results}

From the detector calibration we computed the SLIM acceptance for downgoing IMMs with $g=g_{\mathrm{D}}, 2 g_{\mathrm{D}}, 3 g_{\mathrm{D}}$ and for dyons. For the $i$ th module of area $S_{i}$ the acceptance was computed as

$$
(S \Omega)_{i}=\pi S_{i}\left(1-\frac{1}{p^{2}}\right)
$$
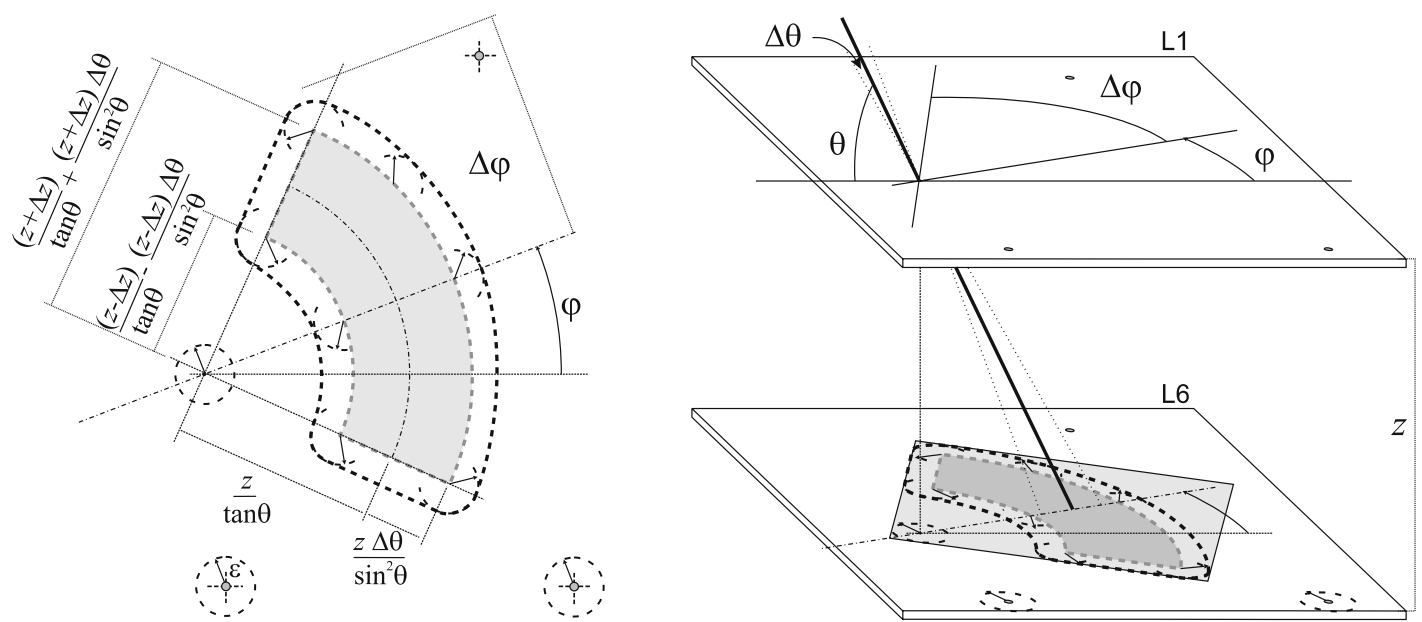

Fig. 6. Illustration of the procedure used to define the "confidence" area where the possible continuation of a candidate track inside two (or more) sheets of the same module was searched for (see text for details) 


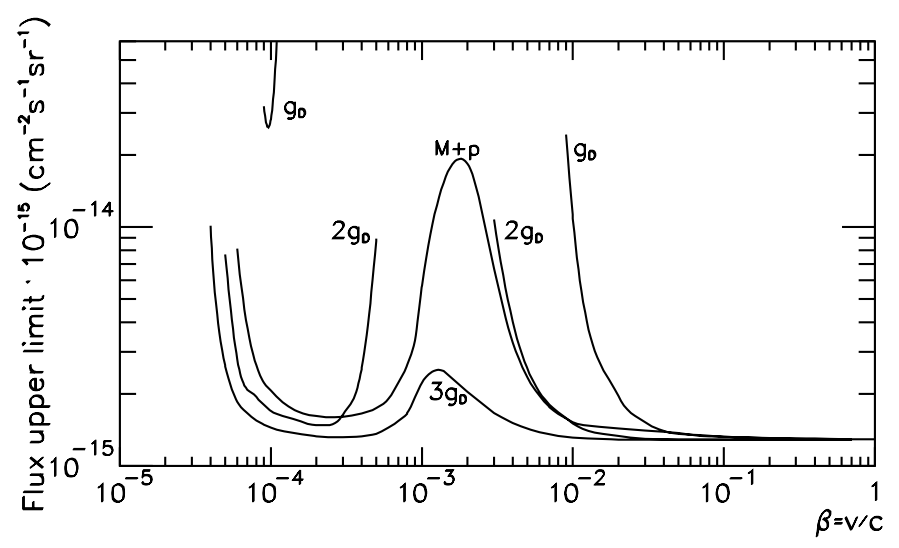

Fig. 7. $90 \%$ C.L. upper limits for a downgoing flux of IMMs with $g=g_{\mathrm{D}}, 2 g_{\mathrm{D}}, 3 g_{\mathrm{D}}$ and for dyons $\left(M+p, g=g_{\mathrm{D}}\right)$ plotted vs. $\beta$ (for strong etching). The poor limits at $\beta \sim 10^{-3}$ arise because the REL is below the threshold (for $g_{\mathrm{D}}$ and $2 g_{\mathrm{D}}$ ) or slightly above the threshold (for $3 g_{\mathrm{D}}$ and dyons), see Sect. 2.3

The total acceptance is the sum of all the individual contributions.

Since no candidates were found, the $90 \%$ C.L. upper limit for a downgoing flux of IMMs and for dyons was computed as

$$
\phi=\frac{2.3}{(S \Omega) \Delta t \epsilon},
$$

where $\Delta t$ is the mean exposure time $(4.22 \mathrm{y}), S \Omega$ is the total acceptance, $\epsilon$ is the scanning efficiency estimated to be $\sim 1$.

The global $90 \%$ C.L. upper limits for the flux of downgoing IMMs and dyons with velocities $\beta>4 \times 10^{-5}$ were computed, as shown in Fig. 7. The flux limit for $\beta>0.03$ is $\sim 1.3 \times 10^{-15} \mathrm{~cm}^{-2} \mathrm{~s}^{-1} \mathrm{sr}^{-1}$.

Two "strange events" were observed and were finally classified as manufacturing defects in a small subset of CR39 NTDs. These "strange events" are discussed in detail elsewhere [48].

\section{Conclusions}

We etched and analyzed $427 \mathrm{~m}^{2}$ of CR39, with an average exposure time of 4.22 years. No candidate passed the search criteria. The $90 \%$ C.L. upper limits for a downgoing flux of fast $(\beta>0.03)$ IMM's coming from above are at the level of $1.3 \times 10^{-15} \mathrm{~cm}^{-2} \mathrm{sr}^{-1} \mathrm{~s}^{-1}$. The complete $\beta$ dependence for MMs with $g=g_{\mathrm{D}}, 2 g_{\mathrm{D}}, 3 g_{\mathrm{D}}$ and for dyons is shown in Fig. 7.

Superheavy GUT magnetic monopoles in the cosmic radiation can traverse the Earth. Therefore the SLIM limit on their flux is one half of the IMM flux: $\phi_{\mathrm{GUT}}<$ $6.5 \times 10^{-16} \mathrm{~cm}^{-2} \mathrm{~s}^{-1} \mathrm{sr}^{-1}$ for $\beta>0.03$ for $g=g_{\mathrm{D}}[14-17]$.

Figure 8 shows the flux upper limits for MMs of charge $g=g_{\mathrm{D}}$ and $\beta>0.05$ vs. monopole mass. Note that the SLIM limit is $1.3 \times 10^{-15} \mathrm{~cm}^{-2} \mathrm{sr}^{-1} \mathrm{~s}^{-1}$ for MM masses smaller than $\sim 5 \times 10^{13} \mathrm{GeV}$ and $0.65 \times 10^{-15} \mathrm{~cm}^{-2} \mathrm{sr}^{-1} \mathrm{~s}^{-1}$

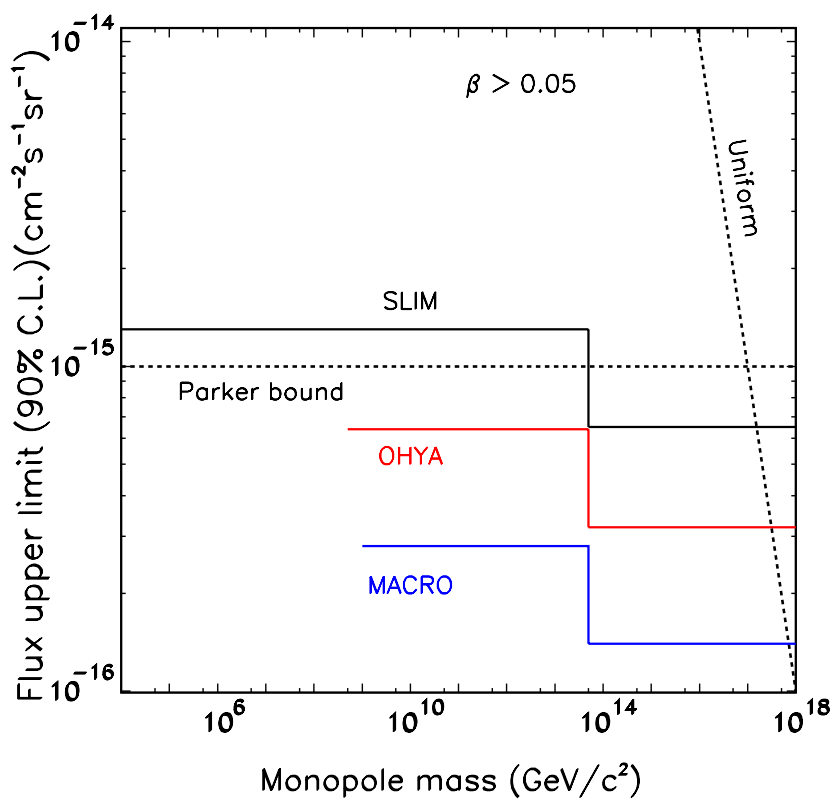

Fig. 8. Flux upper limits for cosmic MMs of charge $g=g_{\mathrm{D}}$ and $\beta>0.05$ vs. monopole mass. The figure shows the $90 \%$ C.L. limits obtained by the SLIM, MACRO [14-17] and OHYA [49] experiments. MMs with masses smaller than $\sim 5 \times 10^{13} \mathrm{GeV}$ are detected only if coming from above; MMs with masses larger than $\sim 5 \times 10^{13} \mathrm{GeV}$ can traverse the Earth, so an isotropic flux is expected. The Parker bound [54], obtained from the survival of the galactic magnetic field, and the limit obtained from the mass density for a uniform density of monopoles in the Universe [55] are also plotted

for masses larger than $\sim 5 \times 10^{13} \mathrm{GeV}$. In Fig. 8 are also shown the limits obtained by the MACRO [14-17] and OHYA [49] experiments for $g=g_{\mathrm{D}}$ magnetic monopoles with $\beta>0.05$.

SLIM is the first experiment to extend the cosmic radiation search for Magnetic Monopoles to masses lower than the GUT scale with a high sensitivity.

The addition of SLIM data to the MACRO data would improve the MACRO limits by only $18 \%$.

Large scale underwater and under ice neutrino telescopes (Amanda, IceCube, ANTARES, NEMO) have the possibility to search for fast IMMs with $\beta>0.5$ to a level lower than the Parker bound $[27,50-53]$.

Acknowledgements. We thank the engineering staff in BNL and in CERN for their help for the heavy ion calibration exposures. We acknowledge the collaboration of our technical staff, in particular L. Degli Esposti, G. Grandi and C. Valieri of INFN Bologna, and the technical staff of the Chacaltaya Laboratory. We thank A. Casoni for typing and correcting the manuscript. We thank INFN and ICTP for providing Grants to non-Italian citizens.

\section{References}

1. P.A.M. Dirac, Proc. R. Soc. London A 133, 60 (1931)

2. G. Giacomelli et al., hep-ex/0005041 
3. M. Bertani et al., Europhys. Lett. 12, 613 (1990)

4. K. Kinoshita et al., Phys. Rev. D 46, R881 (1992)

5. G. Abbiendi et al., hep-ex/0707.0404

6. A. Abulencia et al., Phys. Rev. Lett. 96, 201801 (2006)

7. G.R. Kalbfleisch et al., Phys. Rev. Lett. 85, 5292 (2000)

8. D. Bakari et al., hep-ex/0004019

9. J. Preskill, Ann. Rev. Nucl. Part. Sci. 34, 461 (1984)

10. G. Giacomelli, Riv. Nuovo Cimento 7N12, 1 (1984)

11. G. Giacomelli, Riv. Nuovo Cimento 16N3, 1 (1993)

12. D.E. Groom, Phys. Rep. 140, 323 (1986)

13. G. Giacomelli et al, hep-ex/0702050

14. M. Ambrosio et al., Eur. Phys. J. C 25, 511 (2002)

15. M. Ambrosio et al., Eur. Phys. J. C 26, 163 (2002)

16. M. Ambrosio et al., Astropart. Phys. 18, 27 (2002)

17. M. Ambrosio et al., Nucl. Instrum. Methods A 486, 663 (2002)

18. G. Lazarides, Q. Shafi, Phys. Lett. B 148, 35 (1984)

19. P.H. Frampton, T.W. Kephart, Phys. Rev. D 42, 3892 (1990)

20. T.W. Kephart, Q. Shafi, Phys. Lett. B 520, 313 (2001)

21. T.W. Kephart, T.J. Weiler, Astropart. Phys. 4, 271 (1996)

22. C.O. Escobar, R.A. Vasquez, Astropart. Phys. 10, 197 (1999)

23. D. Bakari et al., hep-ex/0003028 (2000)

24. S. Cecchini et al., Il Nuovo Cimento 24C, 639 (2001)

25. J. Derkaoui et al., Astropart. Phys. 10, 339 (1999)

26. J. Derkaoui et al., Astropart. Phys. 9, 173 (1998)

27. ICECUBE Collaboration, H. Wissing, 30th ICRC (Merida, 2007), p. 139 [astro-ph/0711.0353]

28. A. Witten, Phys. Rev. D 30, 272 (1984)

29. A. De Rujula, S.L. Glashow, Nature 312, 734 (1984)
30. S. Balestra et al., hep-ex/0506075

31. S. Balestra et al., Czech. J. Phys. 56, A221 (2006)

32. S. Balestra et al., hep-ex/0601019

33. S. Cecchini et al., Radiat. Meas. 40, 405 (2005)

34. S. Coleman, Nucl. Phys. B 262, 263 (1985)

35. A. Kusenko et al., Phys. Lett. B 418, 46 (1998)

36. J. Arafune et al., Phys. Rev. D 62, 105013 (2000)

37. S. Cecchini et al., Radiat. Meas. 34, 55 (2001)

38. S. Balestra et al., Nucl. Instrum. Methods B 254, 254 (2007)

39. S. Manzoor et al., Radiat. Meas. 40, 433 (2005)

40. S. Manzoor et al., Nucl. Phys. B Proc. Suppl. 172, 296 (2007)

41. G. Giacomelli et al., Nucl. Instrum. Methods A 411, 41 (1998)

42. H. Schraube et al., Radiat. Prot. Dosim. 84, 309 (1999)

43. A. Zanini et al., Il Nuovo Cimento 24C, 691 (2001)

44. A. Zanini et al., J. Atmosph. Sol.-Terr. Phys. 67, 755 (2005)

45. S. Cecchini et al., nucl-ex/0801.3195, accepted by Nucl. Phys. A

46. A. Noll et al., Nucl. Tracks Radiat Meas. 15, 265 (1988)

47. J. Derkaoui et al., Astropart. Phys. 10, 339 (1999)

48. S. Balestra et al., hep-ex/0802.2056

49. S. Orito et al., Phys. Rev. Lett. 66, 1951 (1991)

50. http://amanda.uci.edu/

51. http://icecube.wisc.edu/

52. http://antares.in2p3.fr/

53. http://nemo.in2p3.fr/

54. M.S. Turner et al., Phys. Rev. D 26, 1296 (1982)

55. A.H. Guth, Phys. Rev. D 23, 347 (1981) 